\title{
Prevalence and Severity of Asthma, Rhinitis, and Atopic Eczema in 13- to 14-Year-Old Schoolchildren from Southern Brazil
}

\author{
Arnaldo C. Porto Neto, MD, Msc; Rafael D’Agostini Annes, MD; Nathalia M.M. Wolff, \\ Andréia P. Klein; Francisco C. dos Santos, MD; Juliana L. Dullius; Marina Gressler; \\ Letícia S. Muller; Cristine F. Angonese, MD; Sérgio Menna-Barreto, MD, PhD
}

\begin{abstract}
The objective of this study was to investigate the prevalence and severity of asthma, rhinitis, and atopic eczema in schoolchildren from southern Brazil. A cross-sectional study was carried out with the International Study of Asthma and Allergies in Childhood phase III written questionnaire. The questionnaire was self-applied by 2,948 randomly selected schoolchildren aged 13 to 14 years. The lifetime prevalence rates of symptoms were as follows: wheezing, $40.8 \%$; rhinitis, $40.7 \%$; eczema, $13.6 \%$; self-reported asthma, $14.6 \%$; rhinitis, $31.4 \%$; eczema, $13.4 \%$. Rhinitis was reported by $55 \%$ of adolescents with current asthma ( $60 \%$ females vs $46.9 \%$ males). Girls 13 to 14 years of age had higher prevalence rates of asthma, rhinitis, and eczema than boys had. Atopic eczema was reported by $42.7 \%$ of girls and $31.4 \%$ of boys with asthma. The prevalence rates were statistically significant for symptoms of asthma, rhinitis, and atopic eczema in females. However, there were no statistically significant differences between the sexes in regard to reported asthma and bronchospasm induced by exercise.
\end{abstract}

Asthma is the most common chronic disease in childhood. ${ }^{1}$ Recent studies suggest that its prevalence is increasing in children and adolescents, as are hospitalization rates for this condition, despite

A.C. Porto Neto-Division of Pediatric AllergyImmunology, São Vicente de Paulo University Hospital Faculty of Medicine, University of Passo Fundo, Passo Fundo, RS, Brazil; R. D’Agostini Annes, N.M.M. Wolff, A.P. Klein, F.C. dos Santos, J.L. Dullius, M. Gressler, L.S. Muller, C.F.Angonese-Faculty of Medicine, University of Passo Fundo, Passo Fundo, RS, Brazil; S. MennaBarreto-Faculty of Medicine, Federal University of Rio Grande do Sul; Pneumology Service, Hospital de Clínicas de Porto Alegre

Correspondence to: Dr. Arnaldo C. Porto Neto, Rua Gabriel Bastos 110, Passo Fundo, RS - Brazil; E-mail: portoneto@brturbo.com.br

DOI 10.2310/7480.2006.00001 considerable advances in knowledge and the development of efficient medications..$^{2-4}$ Asthma has important repercussions, both economic and social, contributing to the overloading of health services., ${ }^{5,6}$

Several hypotheses have been suggested to explain this increase, such as better recognition of the disease, an increasing number of doctors, and environmental factors (namely, changes in lifestyle and urbanization in industrialized countries). It may also be explained by differences between research methods, which implies that epidemiologic studies on asthma need be standardized for temporal and international comparisons.

In 1992, aiming to maximize the value of epidemiologic investigations of asthma and other allergic diseases in childhood and to make international collaboration easier, the International Study of Asthma and Allergies in Childhood (ISAAC) proposed a single method for research. ${ }^{7}$ 
Phase I of ISAAC showed that Brazil occupies eighth place in prevalence, having a mean prevalence of $20 \%$ for current asthma. For ages 13 to 14 years, the prevalence rate varies from 4.8 to $27.1 \% .^{8}$

The aim of this study was to investigate the prevalence of asthma, rhinitis, and atopic eczema and to investigate the relationship between rhinitis and eczema with severe asthma in school-aged adolescents living in the city of Passo Fundo in southern Brazil, an agricultural region, as part of an ISAAC phase III project.

\section{Materials and Methods}

This study was carried out in Passo Fundo, a city located in the state of Rio Grande do Sul (latitude $28^{\circ} \mathrm{S}$ and longitude $52^{\circ} 04^{\prime} \mathrm{W}$ ), which shares borders with Uruguay and Argentina. The majority of the population ( $88.8 \%$ ) is Caucasian, descendants of settlers from Portugal as well as immigrants from Italy, Germany, and other European countries. The total population consists of 182,233 inhabitants, and the annual per capita gross product is approximately $\$ 4,000$ (US). The climate is temperate, and the region has the characteristics of a humid subtropical region with well-distributed rainfall.

The study was a cross-sectional survey of schoolchildren aged 13 to 14 years. The students were selected from children who attended the public and private schools of Passo Fundo. Information regarding the number of schools and students was obtained from the records at the Secretariat of Education. In the year 2002, 5,932 students in this age group were enrolled in public and private schools, distributed among a total of 80 schools. It was determined that schools with 50 or more enrolled students within this age group would be assessed, according to the ISAAC protocol. A total of 46 such schools were identified. From these, 26 schools were chosen by systematic randomized sampling, resulting in a sample of 3,200 students.

\section{Questionnaire}

The instrument used in this study was the ISAAC project pattern written questionnaire (WQ). In Brazil, the questionnaires were translated into Por- tuguese and validated..$^{9-11}$ The only alteration in the questionnaire was a modification of the question on seasonal rhinitis, formulated as "Have you ever had allergic rhinitis," instead of "Have you ever had hay fever," as in the original English version, because the term "hay fever" as a synonym for seasonal allergic rhinitis is little known in Brazil. ${ }^{10}$

WQs were distributed in classrooms and filled out by the adolescents under researchers' supervision from May to September, 2002 (autumn and winter). For absent students, another opportunity was given at a later time. Every WQ that was correctly filled out was considered valid.

\section{Ethical Approval and Consent}

The study was approved by the Ethical Committee and Research of the Hospital das Clínicas de Porto Alegre, Faculty of Medicine, Federal University of Rio Grande do Sul.

Permission for developing the survey in the classrooms was given by the school principals, and consent for participation was provided by parents and by the adolescents themselves.

\section{Statistical Analysis and Power Calculation}

Collected data were entered into a database in double-entry EPI Info version 6.04, provided by the ISAAC coordinators. Statistical analysis was performed with EPI Info version 6.04D. Nonparametric tests (Pearson's chi-square test was used for trends), odds ratio (OR), and 95\% confidence interval $(95 \% \mathrm{CI})$ were used to measure the association force among variables, and a $p$ value of $<.05 \%$ was considered to be significant.

With a sample of 3,000 subjects, the power to detect the difference in the 1-year prevalence of wheezing and severe asthma between two centers would be $99 \%$ and $90 \%$, respectively, at the $1 \%$ level of significance. ${ }^{7}$

\section{Definitions}

In the present study, we defined subjects by the following criteria. Individuals were defined as having had a severe attack of asthma if they answered 
Table 1 Self-Reported Prevalence of Asthma, Rhinitis, and Eczema and Their Related Symptoms in 13- to 14-Year-Old Schoolchildren

\begin{tabular}{|c|c|c|c|c|c|}
\hline Symptom* & $\begin{array}{l}\text { Girls }(\%) \\
(\mathrm{n}=1,513)\end{array}$ & $\begin{array}{l}\text { Boys }(\%) \\
(\mathrm{n}=1,435)\end{array}$ & $\begin{array}{c}\text { All }(\%) \\
(\mathrm{n}=2,948)\end{array}$ & $\begin{array}{c}\text { OR } \\
(95 \% C I)\end{array}$ & $\mathrm{p}$ Value \\
\hline \multicolumn{6}{|l|}{ Asthma } \\
\hline Ever wheezed & 43.6 & 37.8 & $40.8^{\dagger}$ & $1.27(1.09-1.48)$ & $<.01$ \\
\hline Wheeze in the past year & 23.1 & 17.7 & $20.5^{\dagger}$ & $1.40(1.16-1.69)$ & $<.001$ \\
\hline Wheezing with exercise last year & 22.9 & 20.1 & 21.5 & $1.18(0.98-1.41)$ & $>.05$ \\
\hline Persistent cough last year & 47.1 & 31.3 & $39.4^{\dagger}$ & $1.96(1.68-2.29)$ & $<.001$ \\
\hline Ever had asthma & 13.5 & 15.7 & 14.60 & $0.83(0.67-1.03)$ & $>.05$ \\
\hline \multicolumn{6}{|l|}{ Rhinitis } \\
\hline Ever had rhinitis & 47.1 & 34.0 & $40.7^{\dagger}$ & $1.72(1.48-2.01)$ & $<.001$ \\
\hline Rhinitis in the past year & 35.2 & 23.6 & $29.5^{\dagger}$ & $1.76(1.49-2.08)$ & $<.001$ \\
\hline $\begin{array}{l}\text { Associated itchy eye in } \\
\text { the past year }\end{array}$ & 20.8 & 10.7 & $15.9^{\dagger}$ & $2.20(1.78-2.74)$ & $<.001$ \\
\hline Ever had allergic rhinitis & 36.5 & 26.1 & $31.4^{\dagger}$ & $1.63(1.39-1.92)$ & $<.001$ \\
\hline \multicolumn{6}{|l|}{ Eczema } \\
\hline Chronic rash ever & 15.7 & 11.4 & $13.6^{\dagger}$ & $1.44(1.15-1.80)$ & $<.001$ \\
\hline Chronic rash in the past year & 10.5 & 6.7 & $8.6^{\dagger}$ & $1.64(1.24-2.16)$ & $<.001$ \\
\hline $\begin{array}{l}\text { Chronic rash with } \\
\text { typical distribution }\end{array}$ & 6.3 & 3.6 & $5^{\dagger}$ & $1.84(1.28-2.65)$ & $<.001$ \\
\hline Ever had eczema & 15.3 & 11.4 & $13.4^{\dagger}$ & $1.40(1.12-1.74)$ & $<.01$ \\
\hline
\end{tabular}

$\mathrm{CI}=$ confidence interval; $n=$ population size; $\mathrm{OR}=$ odds ratio.

*From written questionnaire.

Indicates significance between girls and boys.

affirmatively to the question, "In the last 12 months, has wheezing ever been severe enough to limit your speech to only one or two words at a time between breaths?" Individuals were defined as having severe asthma if they answered affirmatively to questions asking if they (1) had more than 12 attacks of wheezing in the last year, (2) had disturbed sleep due to wheezing one or more nights per week, (3) had wheezing severe enough to limit their speech to only one or two words between breaths, and (4) had wheezing during or after exercise.

\section{Results}

The questionnaires were answered by students in the classroom, and immediately returned. A total of 3,161 questionnaires were distributed. Of the 3,048 that were returned, $2,948(93.2 \%)$ were correctly filled out. Of the respondents, $51.3 \%$ were female and $48.7 \%$ were male; $55.5 \%$ were 13 years of age and $44.5 \%$ were 14 years of age.
Tables 1 and 2 respectively show the prevalence and severity of asthma, rhinitis, and eczema. The prevalence rate was significantly higher for females than for males $(p<.05)$. "Ever wheezed" and "ever had rhinitis" were the most common affirmative responses, these symptoms respectively having a lifetime prevalence of $43.6 \%$ and $47.1 \%$ in females and $37.8 \%$ and $34.0 \%$ in males.

Females also responded affirmatively more often than males to having had wheezing in the previous 12 months, nocturnal coughing without respiratory infection, symptoms of rhinitis in the previous 12 months, and symptoms of rhinoconjunctivitis $(p<.001)$. Females also presented with more attacks of severe asthma than did males (OR, 2.05; 95\% CI, 1.40-2.99; $p<.001$ ). Regarding the questions on "asthma diagnosed by the doctor" and "wheezing during or after exercise," there was no statistically significant difference between the sexes. Symptoms of eczema were also more frequent among female adolescents $(p<.001)$. Affirmative and concomitant answers 
Table 2 Prevalence of Reported Symptoms Indicating Severity of Asthma, Rhinitis, and Eczema

\begin{tabular}{|c|c|c|c|c|}
\hline Symptom & $\begin{array}{c}\text { Girls }(\%) \\
(\mathrm{n}=1,513)\end{array}$ & $\begin{array}{c}\text { Boys }(\%) \\
(\mathrm{n}=1,435)\end{array}$ & $\begin{array}{c}\text { All (\%) } \\
(\mathrm{n}=2,948)\end{array}$ & $\mathrm{p}$ Value \\
\hline \multicolumn{5}{|l|}{ Wheeze in past year } \\
\hline \multicolumn{5}{|l|}{ Number of wheezing episodes } \\
\hline $1-3$ & $22.5^{*}$ & 16.8 & 19.7 & $<.01$ \\
\hline $4-12$ & 1.3 & 1.7 & 1.5 & $>.05$ \\
\hline$>12$ & 0.1 & $0.6^{*}$ & 0.3 & $<.01$ \\
\hline \multicolumn{5}{|l|}{ Awoken by wheeze } \\
\hline$<1$ time each week & 10.9 & 8.9 & 9.9 & \\
\hline$\geq 1$ time each week & $4.8^{*}$ & 2.6 & 3.8 & $<.01$ \\
\hline Limitation of speech during wheezing & 6.3 & 3.2 & 4.8 & $<.0001$ \\
\hline \multicolumn{5}{|l|}{ Rhinitis in past year } \\
\hline \multicolumn{5}{|l|}{ Interference with daily activity } \\
\hline Little & $18.2 *$ & 11.9 & 15.1 & $<.01$ \\
\hline Moderate & $3.8^{*}$ & 2.4 & 3.2 & $<.05$ \\
\hline Severe & $2 *$ & 0.8 & 1.4 & $<.01$ \\
\hline \multicolumn{5}{|l|}{ Rashes in past year } \\
\hline Persistent rash without clearing & $7.5^{*}$ & 4.8 & 6.2 & .003 \\
\hline \multicolumn{5}{|l|}{ Kept awake by rash } \\
\hline$<1$ time each week & $3.4^{*}$ & 2.4 & 2.9 & $>.05$ \\
\hline$\geq 1$ time each week & $1.9^{*}$ & 0.7 & 1.3 & $<.01$ \\
\hline
\end{tabular}

$\mathrm{n}=$ size of sample.

*Indicates significance between girls and boys.

for asthma, rhinitis, and atopic eczema were given by $2.1 \%$ of the adolescents. Approximately $55 \%$ of the subjects with current asthma reported having current rhinitis (64\% females, compared to $36 \%$ males). Of the subjects who had current asthma, 36\% had associated allergic rhinoconjunctivitis (68\% females, compared to $32 \%$ males). Approximately $10 \%$ of the subjects with current asthma had atopic eczema (72\% females, compared to $28 \%$ males).

Symptoms of current rhinitis and atopic eczema were significantly associated with symptoms of current asthma as well as with severe attacks of asthma (OR for current asthma, 3.84; 95\% CI, 2.67-5.52; $p<.0001$ ) (OR for atopic eczema, 4.0; 95\% CI, 2.62-6.13; $p<.0001)$.

\section{Discussion}

In epidemiologic surveys that use WQs for obtaining study data, it is important to reach a total num- ber of respondents that is close to the estimated population because nonrespondents may generate errors. ${ }^{12}$ It is accepted that a rate of response of about $90 \%$ is required to avoid such deviations in data. ${ }^{7}$ In this study, we observed a rate of about $93.2 \%$, comparable to other studies in which the ISAAC protocol was used.

In the present study, there was a predominance of symptoms of asthma, rhinitis, and atopic eczema in girls. Recent studies indicate that the predominance observed for male preadolescents generally decreases at the end of adolescence, ${ }^{13}$ resulting in an inversion of the proportions between the sexes. ${ }^{14,15}$ One possible explanation for this is that boys in this age group might tend to underestimate their symptoms whereas girls in this age group might tend to overestimate symptoms. ${ }^{15}$ Anatomic $^{16}$ and hormonal factors characteristic of puberty (especially the increase of estradiol levels $)^{17}$ might also explain this change.

The number of wheezing attacks in the past 12 months is the most important item in defining 
cases of asthma because a characteristic of the disease is repeated episodes of wheezing. Children with four or more wheezing attacks in a year have a greater probability of being asthmatic. ${ }^{18}$ In our study, the 12-month prevalence of wheezing (current asthma) for these children was $20.5 \%$. Approximately $90 \%$ of the adolescents with symptoms of current asthma reported one to three wheezing attacks. One in 10 reported having four or more wheezing attacks per year. One-fifth of those who wheezed reported having had frequent sleep disturbances one or more times per week. About onefourth of the adolescents who wheezed reported having limitations of speech during wheezing attacks. These results are similar to those observed by Shamssain and Shamsian, in England. ${ }^{19}$

Epidemiologic studies consistently show that asthma and rhinitis are frequently associated in the same patient. ${ }^{20,21}$ For adolescents aged 13 to 14 years, the ISAAC protocol showed a $3.4 \%$ prevalence of association between asthma and allergic rhinitis, with a variation of 0.5 to $40.0 \% .^{22}$ In this study, approximately $55 \%$ of the adolescents with symptoms of current asthma had current rhinitis. On the other hand, $36.9 \%$ of the subjects with current asthma reported symptoms of rhinoconjunctivitis. This is confirmation that asthma seems to be more frequently associated with perennial rhinitis than with seasonal rhinitis. ${ }^{23}$ Of the adolescents with current rhinitis plus current asthma, $75 \%$ had severe asthma (OR, 7.23; 95\% CI, $1.78-34.09 ; p=.0016)$, indicating that among asthmatic patients, the presence of rhinitis is a risk factor for severe asthma.

Atopic eczema predicts the development of asthma and allergic rhinitis, being the "entrance point" for subsequent allergic diseases. ${ }^{24}$ Approximately $80 \%$ of children with atopic eczema eventually may develop rhinitis or asthma, suggesting a common systemic relation between respiratory allergy and atopic eczema. ${ }^{25}$ In this study, the data show that $25 \%$ of the adolescents with atopic eczema had more acute attacks of asthma (OR, 4.01; 95\% CI, 2.62-6.13; relative risk [RR], $2.51-5.12 ; p<.0001)$ than they had severe asthma (OR, 3.55; $p=.078)$.

The association of asthma, rhinitis, and eczema concomitantly is a reality. Austin and colleagues, using the criteria of the ISAAC protocol in a study involving 27,507 children aged 12 to 14 years, verified that $4 \%$ of the children reported associated symptoms of asthma, rhinitis, and eczema. ${ }^{26}$ Shamssain and Shamsian, assessing 3,000 adolescents aged 13 to 14 years in Sunderland, England, verified the concomitance of asthma, rhinitis, and eczema in $5.1 \%$ of subjects. ${ }^{19}$ Data in the present study showed that $2.1 \%$ of the adolescents presented concomitantly with asthma, rhinitis, and eczema; these values were below those found in the studies conducted in England. When our data are compared with those of other centers at a similar latitude in Latin America, it is observed that there is a higher prevalence of asthma symptoms in Passo Fundo ${ }^{27}$ (Table 3).

Regarding the limitations of the study, the first is the fact that a self-reported questionnaire may be subject to recall bias, and the study did not include any objective tests such as bronchial hyperresponsiveness (BHR) testing or exercise challenge. However, previous work showed that in an English-speaking population, the ISAAC WQ has reasonable sensitivity and specificity for BHR. ${ }^{28}$ In Brazil, Camelo-Nunes and colleagues, by constructive validation of BHR by methacholine testing, showed that the questionnaire option "wheezing in the past 12 months" is the one that has the best discriminatory power in the screening of asthmatic and nonasthmatic patients. ${ }^{29}$

A second limitation of this study was the absence of questions on possible risk factors such as diet, socioeconomic conditions, ${ }^{8}$ family history of atopy, passive or active smoking, the presence of pets, the mother's education level, and exposure to pesticides ${ }^{30}$ and/or insecticides ${ }^{31}$ (all of which, being important for epidemiologic studies of asthma and allergic diseases, will be objects of a subsequent study). In addition, using a questionnaire did not allow us to generate data on skin test reactivity to inhalant allergens.

\section{Conclusions}

The prevalence rate of asthma in the study population was close to the Brazilian mean but greater than that of several centres in Latin America that 


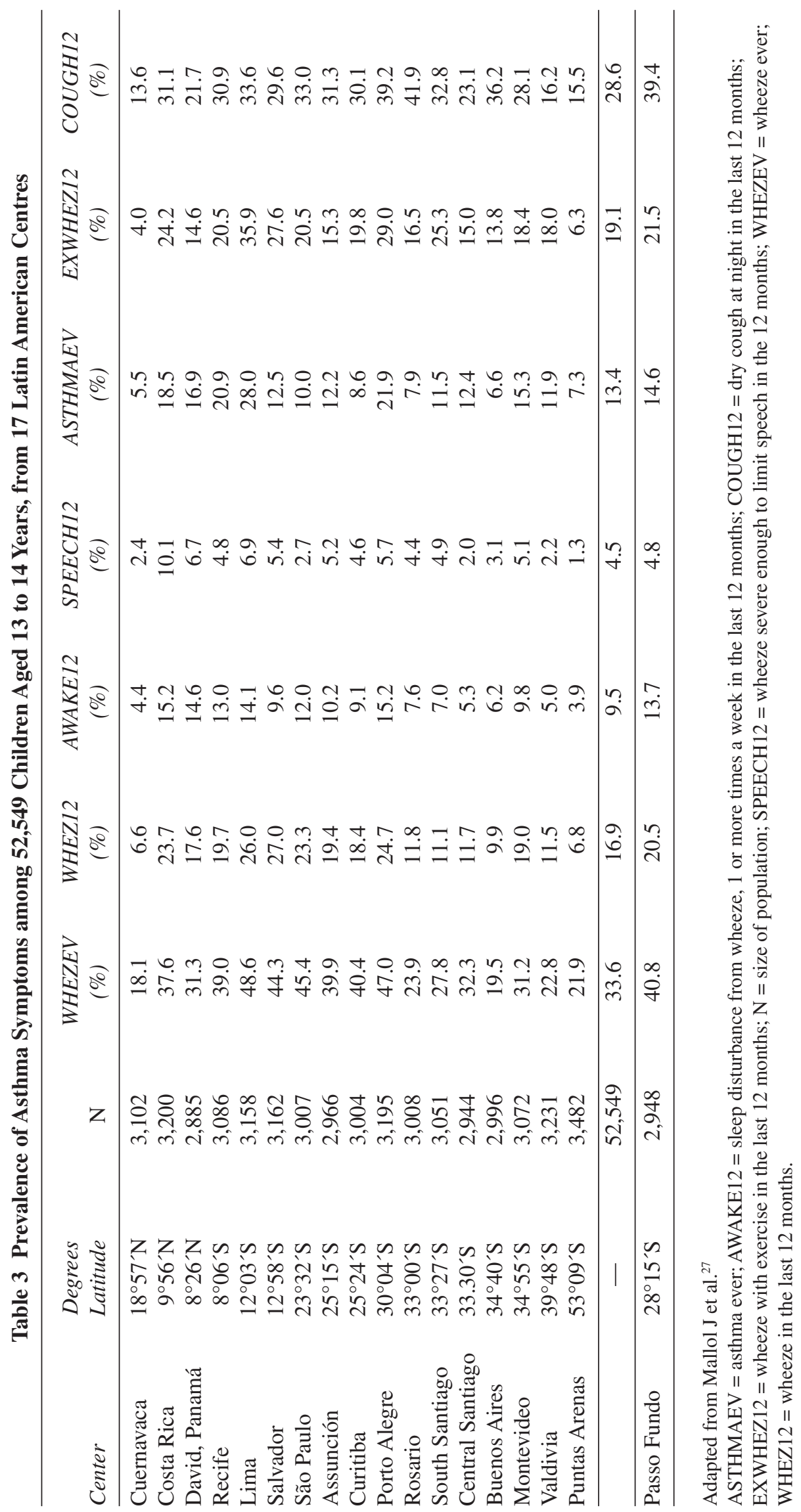


took part in the ISAAC project. Between the sexes, there were no statistically significant differences in regard to diagnosed asthma and exercise-induced bronchospasm; however, there was a predominance of females over males in regard to other symptoms of asthma, rhinitis, and atopic eczema. It was also found that there was a strong association between rhinitis and severe asthma in adolescents; the same was true for atopic eczema and severe attacks of asthma.

Future investigations are needed to confirm these findings, as well as to investigate possible etiologic factors.

\section{Acknowledgements}

We would like to thank the schools and the students who participated. We would also like to thank Dr. Dirceu Solé for his review and suggestions.

\section{References}

1. Myers TK. Pediatrics asthma epidemiology: incidence, morbidity and mortality. Respir Care Clin N Am 2000;6:1-14.

2. European Community Respiratory Health Survey. Variations in the prevalence of respiratory symptoms, self reported asthma attacks, and use of asthma medications in the European Community Respiratory Health Survey (ECRHS). Eur Respir J 1996;9:687-95.

3. Burr ML, Butland BK, King S, VaughanWilliam E. Changes in asthma prevalence: two surveys 15 years apart. Arch Dis Child 1989;64:1452-6.

4. Anderson HR. Increase in hospital admission for childhood asthma: trends in the referral, severity, and readmissions from 1970 to 1985 in a health region of United Kingdom. Thorax 1989;44:614-9.

5. Toelle BG, Peat JK, Melis CM, Woolcock AJ. The cost of childhood asthma to Australian families. Pediatr Pulmonol 1987;19:330-5.

6. Solé D, Camelo-Nunes IC, Wandalsen GF, et al. Is asthma in Brazilian children a public health problem? Rev Bras Alergol Imunopatol 2004;27:185-8.
7. Asher MI, Keil U, Anderson HR, et al. International Study of Asthma and Allergies in Childhood (ISAAC): rationale and methods. Eur Respir J 1995;8:483-91.

8. Solé D, Yamada E, Vana AT, et al. International Study of Asthma and Allergies in Childhood (ISAAC): prevalence of asthma-related symptoms among Brazilian schoolchildren. J Investig Allergol Clin Immunol 2001;11: 123-8.

9. Vanna AT, Yamada E, Arruda LK, et al. International Study of Asthma and Allergies in Childhood: validation of rhinitis symptoms questionnaire and prevalence of rhinitis in schoolchildren in Sao Paulo, Brazil. Pediatr Allergy Immunol 2001;12:95-101.

10. Solé D, Vanna AT, Yamada E, et al. International Study of Asthma and Allergies in childhood (ISAAC) written questionnaire: validation of the asthma component among Brazilian children. J Investig Allergol Immunol 1998;8:376-82.

11. Yamada E, Vanna AT, Naspitz CK, Solé D. International Study of Asthma and Allergies in Childhood (ISAAC) — validation of the written questionnaire (eczema component) and prevalence of atopic eczema among Brazilian children. J Investig Allergol Immunol 2002;12:34-41.

12. Papageorgiou N, Gaga M, Avarlis P, et al. Responses rates influence the estimation of prevalence rates of asthma and asthma-like symptoms. Eur Respir J 1995;8:53s.

13. Pearce N, Weiland S, Keil U, et al. Self-reported prevalence of asthma symptoms in children in Australia, England, Germany and New Zealand: an international comparison using the ISAAC protocol. Eur Respir J 1993;6:1455-61.

14. Sennhauser FH, Kühni CE. Prevalence of respiratory symptoms in Swiss children: is bronchial asthma really more prevalent in boys? Pediatr Pulmonol 1995;19:161-6.

15. Venn A, Lewis $S$, Cooper $M$, et al. Questionnaire study of effect of sex and age on the prevalence of wheeze and asthma in adolescence. BMJ 1998;316:1945-6.

16. Tepper RS, Morgan WJ, Cota K, et al. Physiological growth and development of lung during the first year of life. Am Rev Respir Dis 1986;134:513-9. 
17. Troisi RJ, Speizer FE, Willett WC, et al. Menopause, postmenopausal estrogen preparation and the risk of adult-onset asthma. A prospective cohort study. Am J Respir Crit Care Med 1995;152:1183-8.

18. Reisman JJ, Canny GR, Levison H. Wheezing in infants and young children. In: Tinkelman DG, Naspitz CK, editors. Childhood asthma. New York: Marcel Dekker; 1993. p. 255-82.

19. Shamssain MH, Shamsian N. Prevalence and severity of asthma of asthma, rhinitis, and atopic eczema in 13 to 14 year old schoolchildren from the northeast of England. Ann Allergy Asthma Immunol 2001;86:428-32.

20. Yawn BP, Yunginger JW, Wollan PC, et al. Allergic rhinitis in Rochester, Minnesota, residents with asthma: frequency and impact on health care charges. J Allergy Clin Immunol 1999;103:54-9.

21. Greisner WR, Settipane RJ, Settipane GA. Coexistence of asthma and allergic rhinitis: a 23year follow-up study of college students. Allergy Asthma Proc 1998;19:185-8.

22. Worldwide variation in prevalence of symptoms of asthma, allergic rhinoconjunctivitis, and atopic eczema: ISAAC. The International Study of Asthma and Allergies in Childhood (ISAAC) Steering Committee. Lancet 1998;351: 1225-32.

23. Leynaert B, Bousquet J, Neukirch C, et al. Perennial rhinitis: an independent risk factor for asthma in nonatopic subjects: results from the European Community Respiratory Health Survey. J Allergy Clin Immunol 1999; 104:301-4.
24. Beck LA, Leung DY. Allergen sensitization through the skin induces systemic allergic responses. J Allergy Clin Immunol 2000;106 Suppl 5:S258-63.

25. Leung DYM. Atopic dermatitis: new insights and opportunities for therapeutic intervention. J Allergy Immunol 2000;105:860-76.

26. Austin JB, Kaur B, Anderson HR, et al. Hay fever, eczema and wheeze: a nationwide UK study. Arch Dis Child 1999;81:225-30.

27. Mallol J, Solé D, Asher I, et al. Prevalence of asthma symptoms in Latin America: the International Study of Asthma and Allergies in Childhood (ISAAC). Pediatr Pulmonol 2000;30:439-44.

28. Shaw R, Woodman K, Ayson M, et al. Measuring the prevalence of bronchial hyperresponsiveness in children. Int $\mathrm{J}$ Epidemiol 1995;24:597-602.

29. Camelo-Nunes I, Wandalsen GF, Melo KC, et al. Prevalence of asthma and related symptoms among schoolchildren living in Sao Paulo, Brazil: from 1996 to 1999—study of bronchial responsiveness among adolescents with active asthma and non-asthmatic adolescents"International Study of Asthma and Allergies in Childhood (ISAAC)." Rev Bras Alergol Imunopatol 2001;24:77-89.

30. Salome CM, Marks GB, Savides P, et al. The effect of insecticide aerosols on lung function, airway responsiveness and symptoms in asthmatic subjects. Eur Respir J 2000;16:38-43.

31. Salameh PR, Baldi I, Brochard P, et al. Respiratory symptoms in children and exposure to pesticides. Eur Respir J 2003;22:507-12. 